\title{
Independent sets on the Towers of Hanoi graphs*
}

\author{
Hanlin Chen, Renfang Wu, Guihua Huang, Hanyuan Deng ${ }^{\dagger}$ \\ College of Mathematics and Computer Science, Hunan Normal University, \\ Changsha, Hunan 410081, P. R. China
}

Received 24 December 2014, accepted 16 May 2016, published online 7 December 2016

\begin{abstract}
The number of independent sets is equivalent to the partition function of the hardcore lattice gas model with nearest-neighbor exclusion and unit activity. In this article, we mainly study the number of independent sets $i\left(H_{n}\right)$ on the Tower of Hanoi graph $H_{n}$ at stage $n$, and derive the recursion relations for the numbers of independent sets. Upper and lower bounds for the asymptotic growth constant $\mu$ on the Towers of Hanoi graphs are derived in terms of the numbers at a certain stage, where $\mu=\lim _{v \rightarrow \infty} \frac{\ln i(G)}{v(G)}$ and $v(G)$ is the number of vertices in a graph $G$. Furthermore, we also consider the number of independent sets on the Sierpiński graphs which contain the Towers of Hanoi graphs as a special case.
\end{abstract}

Keywords: Independent sets, the Tower of Hanoi graph, Sierpinski graph, recursion relation, asymptotic growth constant, asymptotic enumeration.

Math. Subj. Class.: 05C30, 05C69

\section{Introduction}

Counting sets satisfying a fixed property in graphs ranges among the classical tasks of combinatorics. There is a vast amount of literatures on this kind of combinatorial problems for various classes of graphs, especially for Sierpiński graphs, by different authors. We note that the set counting problems such as the number of independent sets and the number of matchings have been studied in the past [2, 4, 9, 10, 11, 26, 35, 36].

On one hand, all these graph invariants reflect the structure of a graph in some way, and therefore, some of them are even of interest in theoretical chemistry for the study of molecular graphs (see [32, 38]). For example, the number of independent sets is called

\footnotetext{
* Project supported by the National Natural Science Foundation of China (11401192) and Hunan Provincial Innovation Foundation for Postgraduate (CX2015B162).

${ }^{\dagger}$ Corresponding Author.

E-mail address: hydeng@hunnu.edu.cn (Hanyuan Deng)
} 
Merrifield-Simmons index, the number of matchings is known as Hosoya index in chemistry. It was shown that both correlate well with physicochemical properties of the corresponding molecules (see [23, 30]).

On the other hand, the number of independent sets is equivalent to the partition function of the hard-core lattice gas model with nearest-neighbor exclusion and unit activity. The lattice gas with repulsive pair interaction is an important model in statistical mechanics $[3,13,16,33]$. For the special case with hard-core nearest-neighbor exclusion such that each site can be occupied by at most one particle and no pair of adjacent sites can be simultaneously occupied, the partition function of the lattice gas coincides with the independence polynomial in combinatorics [14,34]. This model is a problem of interest in mathematics $[39,15,24]$. The growth of the number of independent sets in the $m \times n$ grid graph is of interest in statistical physics (see [1]). It is known that the number of independent sets in the $m \times n$ grid graph grows with $\alpha^{m n}$, where $\alpha=1.503048082 \cdots$ is the so-called hard square entropy constant. The bound for this constant was successively improved by Weber [40], Engel [9] and Calkin and Wilf [4].

The number of independent sets and its bounds had been considered on various graphs $[27,29,41]$. It is of interest to consider independent sets on self-similar fractal lattices which have scaling invariance rather than translational invariance [35]. The recursion relations for the numbers of independent sets on the Sierpiński gasket were derived by Chang, Chen and Yan [6]. A special type of self-similar graph that has been of interest is the Hanoi graph, which has been extensively studied in several contexts $[5,7,8,12,17,18,19,20$, 22, 25, 28, 31]. This graph, which is also known as the Tower of Hanoi graph, came from the well known Tower of Hanoi puzzle, as the graph is associated to the allowed moves in this puzzle. We shall derive the recursion relations for the numbers of independent sets on the Towers of Hanoi graphs. Upper and lower bounds for the asymptotic growth constant $\mu$ on the Tower of Hanoi graphs are derived in terms of the numbers at a certain stage, where $\mu=\lim _{v \rightarrow \infty} \frac{\ln i(G)}{v(G)}, i(G)$ and $v(G)$ are the number of independent sets and the number of vertices in a graph $G$, respectively. Furthermore, we also consider the Sierpiński graphs which include the Towers of Hanoi graphs as a special case.

\section{Preliminaries}

We recall some basic definitions about graphs. A graph $G=(V, E)$ with vertex set $V$ and edge set $E$ is always supposed to be undirected, without loops or multiple edges. Vertices $x$ and $y$ are adjacent if $x y$ is an edge in $E$. Let $v(G)=|V|$ be the number of vertices and $e(G)=|E|$ the number of edges in $G$. An independent set is a subset of the vertices such that any two of them are not adjacent. When the number $i(G)$ of independent sets in $G$ grows exponentially with $v(G)$ as $v(G) \rightarrow \infty$, let us define a constant $\mu$ describing this exponential growth:

$$
\mu=\lim _{v(G) \rightarrow \infty} \frac{\ln i(G)}{v(G)} .
$$

We will see that the limit exists for the Towers of Hanoi graphs and some other Sierpiński graphs considered in this paper.

There are many different approaches to construct self-similar graphs. A construction that is specifically geared to be used in the context of enumeration was described in [35], it is no restated and we will also make use of it here. Some examples can be seen in [37].

The Tower of Hanoi graph (or the Hanoi graph), invented in 1883 by the French math- 

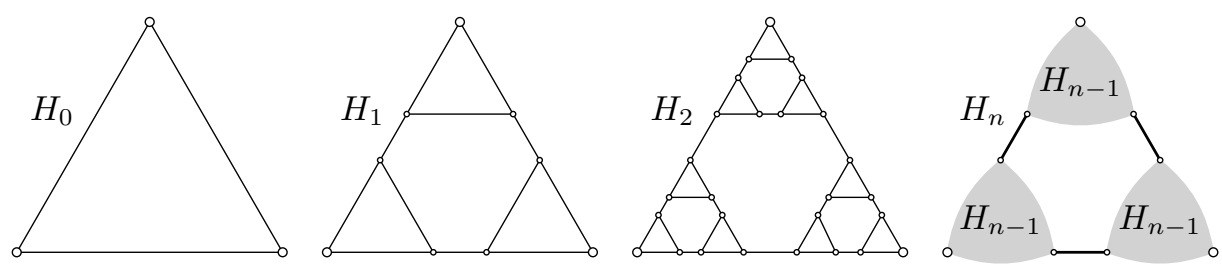

Figure 1: The Towers of Hanoi graphs $H_{0}, H_{1}, H_{2}$ and the construction of $H_{n}$.

ematician Edouard Lucas, has become a classic example in the analysis of algorithms and discrete mathematical structures. There exists an abundant literature on the properties of the Hanoi graph, which includes the study of shortest paths, average eccentricity, to name a few, see [21] and references therein. The Hanoi graph $H_{n}$ is derived from the Tower of Hanoi puzzle with $n$ discs. The vertices of the graph $H_{n}$ in this sequence correspond to all possible configurations of the game Tower with $n+1$ disks and three rods, whereas the edges describe transitions between configurations, see [17], and these graphs are finite Schreier graphs of the Hanoi tower group in [12]. Note that the Tower of Hanoi graph can be constructed by the following recursive-modular method. For $n=0, H_{0}$ is the complete graph $K_{3}$ (also called a 3-clique or triangle). For $n \geq 1, H_{n}$ is obtained from three copies of $H_{n-1}$ joined by three new edges, each one connecting a pair of vertices from two different replicas of $H_{n-1}$, as show in Figure 1. From the construction rule, we can find that the number of vertices of $H_{n}$ is $3^{n+1}$ while the number of edges is $\frac{3^{n+2}-3}{2}$.

\section{The number of independent sets on $H_{n}$}

In this section, we will derive the asymptotic growth constant for the number of independent sets on the Tower of Hanoi graph $H_{n}$ in detail.

For the Tower of Hanoi graph $H_{n}, i_{n}$ is its number of independent sets, $f_{n}$ is its number of independent sets such that all three outmost vertices are not in the vertex subset, $g_{n}$ is its number of independent sets such that only one specified vertex of three outmost vertices is in the vertex subset, $h_{n}$ is its number of independent sets such that exact two specified vertices of the three outmost vertices are in the vertex subset, $p_{n}$ is its number of independent sets such that all three outmost vertices are in the vertex subset. They are illustrated in Figures 2-5, where only the outmost vertices are shown and a solid circle is in the independent set and an open circle is not. Because of rotational symmetry, there are three possible $g_{n}$ and three possible $h_{n}$ such that

$$
i_{n}=f_{n}+3 g_{n}+3 h_{n}+p_{n}
$$

and $f_{0}=g_{0}=1, h_{0}=p_{0}=0, i_{0}=f_{0}+3 g_{0}+3 h_{0}+p_{0}=4$. 
Lemma 3.1. For any nonnegative integer $n$, we have

$$
\begin{aligned}
f_{n+1}= & f_{n}^{3}+6 f_{n}^{2} g_{n}+3 f_{n}^{2} h_{n}+9 f_{n} g_{n}^{2}+6 f_{n} g_{n} h_{n}+2 g_{n}^{3}, \\
g_{n+1}= & f_{n}^{2} g_{n}+2 f_{n}^{2} h_{n}+f_{n}^{2} p_{n}+4 f_{n} g_{n}^{2}+8 f_{n} g_{n} h_{n}+2 f_{n} g_{n} p_{n}+2 f_{n} h_{n}^{2}+3 g_{n}^{3} \\
& +4 g_{n}^{2} h_{n}, \\
h_{n+1}= & f_{n} g_{n}^{2}+4 f_{n} g_{n} h_{n}+2 f_{n} g_{n} p_{n}+3 f_{n} h_{n}^{2}+2 f_{n} h_{n} p_{n}+2 g_{n}^{3}+7 g_{n}^{2} h_{n}+2 g_{n}^{2} p_{n} \\
& +4 g_{n} h_{n}^{2}, \\
p_{n+1}= & g_{n}^{3}+6 g_{n}^{2} h_{n}+3 g_{n}^{2} p_{n}+9 g_{n} h_{n}^{2}+6 g_{n} h_{n} p_{n}+2 h_{n}^{3} .
\end{aligned}
$$

Proof. Note that the number $f_{n+1}$ consists of (i) one configuration where all three $H_{n}$ belong to the class that enumerated by $f_{n}$; (ii) six configurations where one of the $H_{n}$ belongs to the class that enumerated by $g_{n}$ and the other two belong to the class that enumerated by $f_{n}$; (iii) three configurations where one of the $H_{n}$ belongs to the class that enumerated by $h_{n}$ and the other two belong to the class that enumerated by $f_{n}$; (iv) nine configurations where one of the $H_{n}$ belongs to the class that enumerated by $f_{n}$ and the other two belong to the class that enumerated by $g_{n}$; (v) six configurations where all three $H_{n}$ belong to the class that enumerated by $f_{n}, g_{n}$ and $h_{n}$, respectively; (vi) two configurations where all three $H_{n}$ belong to the class that enumerated by $g_{n}$ as illustrated in Figure 2. And

$$
f_{n+1}=f_{n}^{3}+6 f_{n}^{2} g_{n}+3 f_{n}^{2} h_{n}+9 f_{n} g_{n}^{2}+6 f_{n} g_{n} h_{n}+2 g_{n}^{3}
$$

is verified by adding these configurations.

Similarly, the expressions of $g_{n+1}, h_{n+1}$ and $p_{n+1}$ can be obtained with appropriate configurations of its three $H_{n}$ as illustrated in Figures 3-5.

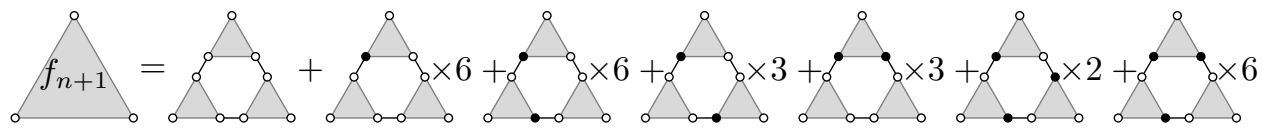

Figure 2: Illustration for the expression of $f_{n+1}$. The multiplication of three on the righthand-side corresponds to the three possible orientations of $H_{n+1}$, the multiplication of two on the right-hand-side corresponds to reflection symmetry with respect to the central vertical axis and the multiplication of six on the right-hand-side corresponds to the six possible of considering both orientations and reflection symmetry.

In the following, we will estimate the value $\mu=\lim _{v \rightarrow \infty} \frac{\ln i\left(H_{n}\right)}{v\left(H_{n}\right)}$ of the asymptotic growth constant for the Tower of Hanoi graph $H_{n}$. The values of $f_{n}, g_{n}, h_{n}, p_{n}$ for small $n$ are listed in Table 1 by Lemma 3.1, and grow exponentially. For the Tower of Hanoi graph $H_{n}$, define the ratios

$$
\alpha_{n}=\frac{g_{n}}{f_{n}}, \quad \beta_{n}=\frac{h_{n}}{g_{n}}, \quad \gamma_{n}=\frac{p_{n}}{h_{n}}
$$

where $n$ is a positive integer. Their values for small $n$ are listed in Table 2. From the initial values of $f_{n}, g_{n}, h_{n}, p_{n}$, it is easy to see that $f_{n}>g_{n}>h_{n}>p_{n}$ for all positive integer $n$ by induction. Alternatively, these inequalities can be obtained by an injection. For instance, if one of the independent sets enumerated by $g_{n}$ is given, one can remove 


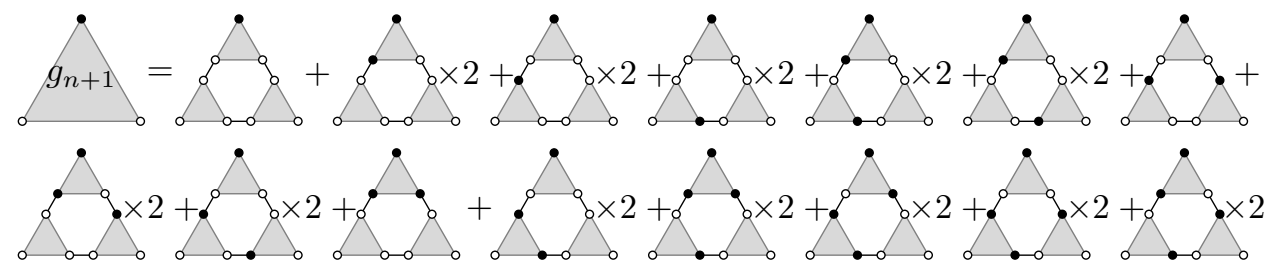

Figure 3: Illustration for the expression of $g_{n+1}$. The multiplication of two on the righthand-side are corresponds to the reflection symmetry with respect to the central vertical axis.

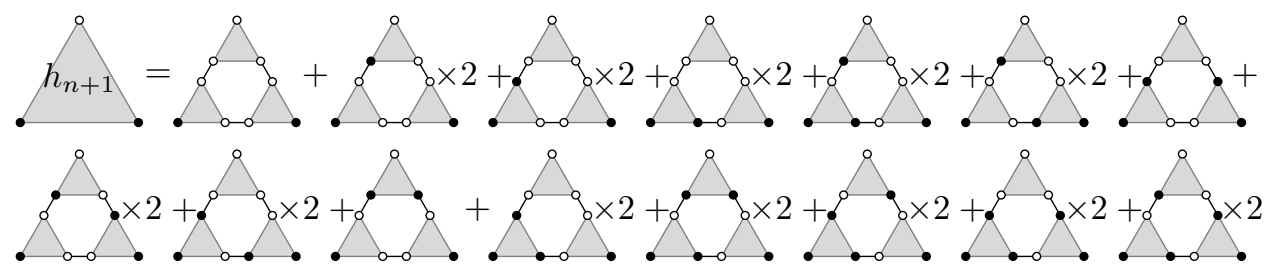

Figure 4: Illustration for the expression of $h_{n+1}$. The multiplication of two on the righthand-side are corresponds to the reflection symmetry with respect to the central vertical axis.

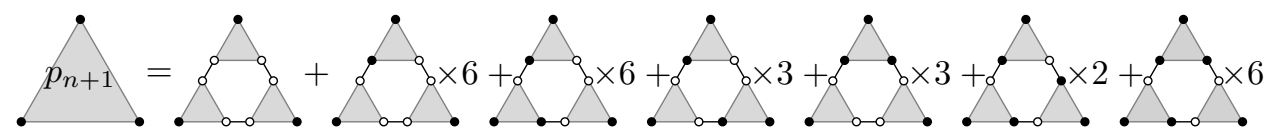

Figure 5: Illustration for the expression of $p_{n+1}$. The multiplication of three on the righthand-side corresponds to the three possible orientations of $H_{n+1}$, the multiplication of two on the right-hand-side corresponds to reflection symmetry with respect to the central vertical axis and the multiplication of six on the right-hand-side corresponds to the six possible of considering both orientations and reflection symmetry.

Table 1: The first few values of $f_{n}, g_{n}, h_{n}, p_{n}$ and $i_{n}$ on $H_{n}$.

\begin{tabular}{rrrrr}
\hline$n$ & 0 & 1 & 2 & 3 \\
\hline$f_{n}$ & 1 & 18 & 38284 & 342408411795232 \\
$g_{n}$ & 1 & 8 & 15840 & 141595222762112 \\
$h_{n}$ & 0 & 3 & 6546 & 58553484583728 \\
$p_{n}$ & 0 & 1 & 2702 & 24213460330512 \\
$i_{n}$ & 4 & 52 & 108144 & 967067994163264 \\
\hline
\end{tabular}

the corner vertex to obtain another independent set that are enumerated by $f_{n}$ such that $f_{n}>g_{n}$ is established. Similarly, other two inequalities can be established. It follows that $\alpha_{n}, \beta_{n}, \gamma_{n} \in(0,1)$. 
Table 2: The first few values of $\alpha_{n}, \beta_{n}, \gamma_{n}$ on $H_{n}$.

\begin{tabular}{rrrr}
\hline$n$ & 1 & 2 & 3 \\
\hline$\alpha_{n}$ & 0.444444444444444 & 0.413749869397137 & 0.413527290465016 \\
$\beta_{n}$ & 0.375 & 0.413257575757575 & 0.413527260606109 \\
$\gamma_{n}$ & 0.333333333333333 & 0.412771157959058 & 0.413527230747269 \\
\hline
\end{tabular}

Lemma 3.2. For any positive integer $n$, the ratios satisfy

$$
\alpha_{n}>\beta_{n}>\gamma_{n}
$$

When $n$ increases, the ratio $\alpha_{n}$ decreases monotonically while $\gamma_{n}$ increases monotonically. The three ratios in the large $n$ limit are equal to each other

$$
\lim _{n \rightarrow \infty} \alpha_{n}=\lim _{n \rightarrow \infty} \beta_{n}=\lim _{n \rightarrow \infty} \gamma_{n}
$$

Proof. By the definition of $\alpha_{n}, \beta_{n}, \gamma_{n}$, we have

$$
\alpha_{n+1}=\alpha_{n} \frac{B_{n}}{A_{n}}, \beta_{n+1}=\alpha_{n} \frac{C_{n}}{B_{n}}, \gamma_{n+1}=\alpha_{n} \frac{D_{n}}{C_{n}}
$$

for a positive integer $n$, where

$$
\begin{aligned}
& A_{n}=1+6 \alpha_{n}+3 \alpha_{n} \beta_{n}+9 \alpha_{n}^{2}+6 \alpha_{n}^{2} \beta_{n}+2 \alpha_{n}^{3}, \\
& B_{n}=1+2 \beta_{n}+\beta_{n} \gamma_{n}+4 \alpha_{n}+8 \alpha_{n} \beta_{n}+2 \alpha_{n} \beta_{n} \gamma_{n}+2 \alpha_{n} \beta_{n}^{2}+3 \alpha_{n}^{2}+4 \alpha_{n}^{2} \beta_{n}, \\
& C_{n}=1+4 \beta_{n}+2 \beta_{n} \gamma_{n}+3 \beta_{n}^{2}+2 \beta_{n}^{2} \gamma_{n}+2 \alpha_{n}+7 \alpha_{n} \beta_{n}+2 \alpha_{n} \beta_{n} \gamma_{n}+4 \alpha_{n} \beta_{n}^{2}, \\
& D_{n}=1+6 \beta_{n}+3 \beta_{n} \gamma_{n}+9 \beta_{n}^{2}+6 \beta_{n}^{2} \gamma_{n}+2 \beta_{n}^{3} .
\end{aligned}
$$

In the following, we show that $\frac{1}{3} \leq \gamma_{n}<\beta_{n}<\alpha_{n} \leq \frac{4}{9}$ by induction on $n$. It is true for $n=1,2,3,4$ from Table 2. Suppose that $\frac{1}{3} \leq \gamma_{n}<\beta_{n}<\alpha_{n} \leq \frac{4}{9}$ for $n \geq 4$.

Let $\varepsilon_{n}=\alpha_{n}-\gamma_{n}$. Then $\varepsilon_{n}>\alpha_{n}-\beta_{n}, \varepsilon_{n}>\beta_{n}-\gamma_{n}$ and $\varepsilon_{n} \in\left(0, \frac{1}{9}\right)$. Now,

$$
\begin{aligned}
\alpha_{n}-\alpha_{n+1}= & \alpha_{n}-\alpha_{n} \frac{B_{n}}{A_{n}}=\frac{\alpha_{n}\left(A_{n}-B_{n}\right)}{A_{n}} \\
= & \frac{\alpha_{n}}{A_{n}}\left[\left(2+6 \alpha_{n}+4 \alpha_{n} \beta_{n}+2 \alpha_{n}^{2}+\beta_{n}\right)\left(\alpha_{n}-\beta_{n}\right)\right. \\
& \left.+\left(2 \alpha_{n} \beta_{n}+\beta_{n}\right)\left(\beta_{n}-\gamma_{n}\right)\right]>0, \\
\alpha_{n+1}-\beta_{n+1}= & \frac{\alpha_{n}\left(B_{n}^{2}-A_{n} C_{n}\right)}{A_{n} B_{n}}>0,
\end{aligned}
$$

where

$$
\begin{aligned}
B_{n}^{2}-A_{n} C_{n}= & \left(10 \alpha_{n}^{2} \beta_{n}+5 \alpha_{n}^{2}+\alpha_{n} \beta_{n}+4 \alpha_{n}+\beta_{n}^{2}+1\right)\left(\alpha_{n}-\beta_{n}\right)^{2}+\left(4 \alpha_{n}^{2} \beta_{n}^{2}+2 \alpha_{n} \beta_{n}^{2}\right. \\
& \left.+6 \alpha_{n} \beta_{n}+2 \beta_{n}\right)\left(\alpha_{n}-\beta_{n}\right)\left(\alpha_{n}-\gamma_{n}\right)+\left(4 \alpha_{n}^{3} \beta_{n}+10 \alpha_{n}^{2} \beta_{n}+2 \alpha_{n} \beta_{n}^{2}\right. \\
& \left.+2 \alpha_{n} \beta_{n}+\beta_{n}^{2}\right)\left(\beta_{n}-\gamma_{n}\right)\left(\alpha_{n}-\beta_{n}\right)+\left(2 \alpha_{n} \beta_{n}^{2}+\beta_{n}^{2}\right)\left(\alpha_{n}-\gamma_{n}\right)\left(\beta_{n}-\gamma_{n}\right) \\
& +\left(4 \alpha_{n}^{2} \beta_{n}^{2}+2 \alpha_{n} \beta_{n}^{2}\right)\left(\beta_{n}-\gamma_{n}\right)^{2}>0,
\end{aligned}
$$




$$
\begin{aligned}
A_{n} B_{n}= & 10 \alpha_{n}+2 \beta_{n}+23 \alpha_{n} \beta_{n}+\beta_{n} \gamma_{n}+8 \alpha_{n} \beta_{n}^{2}+88 \alpha_{n}^{2} \beta_{n}+133 \alpha_{n}^{3} \beta_{n}+70 \alpha_{n}^{4} \beta_{n} \\
& +8 \alpha_{n}^{5} \beta_{n}+36 \alpha_{n}^{2}+56 \alpha_{n}^{3}+35 \alpha_{n}^{4}+6 \alpha_{n}^{5}+48 \alpha_{n}^{2} \beta_{n}^{2}+6 \alpha_{n}^{2} \beta_{n}^{3}+78 \alpha_{n}^{3} \beta_{n}^{2} \\
& +12 \alpha_{n}^{3} \beta_{n}^{3}+12 \alpha_{n}^{3} \beta_{n}^{2} \gamma_{n}+28 \alpha_{n}^{4} \beta_{n}^{2}+12 \alpha_{n}^{2} \beta_{n}^{2} \gamma_{n}+8 \alpha_{n} \beta_{n} \gamma_{n}+3 \alpha_{n} \beta_{n}^{2} \gamma_{n} \\
& +21 \alpha_{n}^{2} \beta_{n} \gamma_{n}+20 \alpha_{n}^{3} \beta_{n} \gamma_{n}+4 \alpha_{n}^{4} \beta_{n} \gamma_{n}+1 \\
> & 4 \alpha_{n}^{4} \beta_{n}+8 \alpha_{n}^{3} \beta_{n}^{2}+20 \alpha_{n}^{3} \beta_{n}+5 \alpha_{n}^{3}+8 \alpha_{n}^{2} \beta_{n}^{2}+9 \alpha_{n}^{2} \beta_{n}+4 \alpha_{n}^{2}+3 \alpha_{n} \beta_{n}^{2} \\
& +2 \alpha_{n} \beta_{n}+\alpha_{n} .
\end{aligned}
$$

Then

$$
\begin{aligned}
\alpha_{n+1}-\beta_{n+1}= & \frac{\alpha_{n}\left(B_{n}^{2}-A_{n} C_{n}\right)}{A_{n} B_{n}} \\
< & \frac{\varepsilon_{n}^{2}}{A_{n} B_{n}}\left[4 \alpha_{n}^{4} \beta_{n}+8 \alpha_{n}^{3} \beta_{n}^{2}+20 \alpha_{n}^{3} \beta_{n}+5 \alpha_{n}^{3}+8 \alpha_{n}^{2} \beta_{n}^{2}+9 \alpha_{n}^{2} \beta_{n}+4 \alpha_{n}^{2}\right. \\
& \left.+3 \alpha_{n} \beta_{n}^{2}+2 \alpha_{n} \beta_{n}+\alpha_{n}\right] \\
< & \varepsilon_{n}^{2}
\end{aligned}
$$

since $\varepsilon_{n}>\alpha_{n}-\beta_{n}$ and $\varepsilon_{n}>\beta_{n}-\gamma_{n}$.

Similarly, we have $\beta_{n+1}-\gamma_{n+1}=\frac{\alpha_{n}\left(C_{n}^{2}-B_{n} D_{n}\right)}{B_{n} C_{n}}>0$, where

$$
\begin{aligned}
C_{n}^{2}-B_{n} D_{n}= & {\left[\left(10 \beta_{n}^{3}+4 \beta_{n}^{2} \gamma_{n}^{2}+4 \beta_{n}^{2}+4 \beta_{n}+1\right)\left(\alpha_{n}-\beta_{n}\right)+\left(2 \beta_{n}^{3}+9 \beta_{n}^{2}\right.\right.} \\
& \left.\left.+2 \beta_{n}\right)\left(\alpha_{n}-\gamma_{n}\right)+\left(2 \alpha_{n} \beta_{n}^{2}+\alpha_{n} \beta_{n}\right)\left(\beta_{n}-\gamma_{n}\right)\right]\left(\alpha_{n}-\beta_{n}\right) \\
& +\left[\left(4 \alpha_{n} \beta_{n}^{3}+10 \beta_{n}^{3}\right)\left(\alpha_{n}-\beta_{n}\right)+\left(4 \alpha_{n} \beta_{n}^{3}+2 \beta_{n}^{3}\right)\left(\alpha_{n}-\gamma_{n}\right)\right. \\
& \left.+\left(2 \alpha_{n} \beta_{n}^{2}+\beta_{n}^{2}\right)\left(\beta_{n}-\gamma_{n}\right)\right]\left(\beta_{n}-\gamma_{n}\right)>0
\end{aligned}
$$

$$
\begin{aligned}
B_{n} C_{n}= & 16 \alpha_{n}^{3} \beta_{n}^{3}+8 \alpha_{n}^{3} \beta_{n}^{2} \gamma_{n}+40 \alpha_{n}^{3} \beta_{n}^{2}+6 \alpha_{n}^{3} \beta_{n} \gamma_{n}+29 \alpha_{n}^{3} \beta_{n}+6 \alpha_{n}^{3}+8 \alpha_{n}^{2} \beta_{n}^{4} \\
& +20 \alpha_{n}^{2} \beta_{n}^{3} \gamma_{n}+58 \alpha_{n}^{2} \beta_{n}^{3}+4 \alpha_{n}^{2} \beta_{n}^{2} \gamma_{n}^{2}+44 \alpha_{n}^{2} \beta_{n}^{2} \gamma_{n}+101 \alpha_{n}^{2} \beta_{n}^{2}+18 \alpha_{n}^{2} \beta_{n} \gamma_{n} \\
& +60 \alpha_{n}^{2} \beta_{n}+11 \alpha_{n}^{2}+4 \alpha_{n} \beta_{n}^{4} \gamma_{n}+6 \alpha_{n} \beta_{n}^{4}+4 \alpha_{n} \beta_{n}^{3} \gamma_{n}^{2}+30 \alpha_{n} \beta_{n}^{3} \gamma_{n}+40 \alpha_{n} \beta_{n}^{3} \\
& +6 \alpha_{n} \beta_{n}^{2} \gamma_{n}^{2}+43 \alpha_{n} \beta_{n}^{2} \gamma_{n}+64 \alpha_{n} \beta_{n}^{2}+14 \alpha_{n} \beta_{n} \gamma_{n}+35 \alpha_{n} \beta_{n}+6 \alpha_{n}+2 \beta_{n}^{3} \gamma_{n}^{2} \\
& +7 \beta_{n}^{3} \gamma_{n}+6 \beta_{n}^{3}+2 \beta_{n}^{2} \gamma_{n}^{2}+10 \beta_{n}^{2} \gamma_{n}+11 \beta_{n}^{2}+3 \beta_{n} \gamma_{n}+6 \beta_{n}+1 .
\end{aligned}
$$

Thus, we have

$$
\begin{aligned}
\beta_{n+1}-\gamma_{n+1}= & \frac{\alpha_{n}\left(C_{n}^{2}-B_{n} D_{n}\right)}{B_{n} C_{n}} \\
< & \frac{\varepsilon_{n}^{2}}{B_{n} C_{n}}\left[8 \alpha_{n}^{2} \beta_{n}^{3}+4 \alpha_{n}^{2} \beta_{n}^{2}+\alpha_{n}^{2} \beta_{n}+24 \alpha_{n} \beta_{n}^{3}+4 \alpha_{n} \beta_{n}^{2} \gamma_{n}^{2}+14 \alpha_{n} \beta_{n}^{2}\right. \\
& \left.+6 \alpha_{n} \beta_{n}+\alpha_{n}\right]<\varepsilon_{n}^{2} .
\end{aligned}
$$


And

$$
\begin{aligned}
\gamma_{n+1}-\gamma_{n}= & \frac{1}{C_{n}}\left(\alpha_{n} D_{n}-\gamma_{n} C_{n}\right) \\
= & \frac{1}{C_{n}}\left[\left(1+4 \beta_{n}+2 \beta_{n}^{2}+2 \beta_{n}^{2} \gamma_{n}\right)\left(\alpha_{n}-\gamma_{n}\right)+\left(2 \alpha_{n}+7 \alpha_{n} \beta_{n}+2 \beta_{n} \gamma_{n}\right.\right. \\
& \left.\left.+2 \alpha_{n} \beta_{n} \gamma_{n}+2 \alpha_{n} \beta_{n}^{2}\right)\left(\beta_{n}-\gamma_{n}\right)+3 \beta_{n} \gamma_{n}\left(\alpha_{n}-\beta_{n}\right)\right]>0 .
\end{aligned}
$$

So, we have (i) $\alpha_{n}-\alpha_{n+1}>0$, (ii) $0<\alpha_{n+1}-\beta_{n+1}<\varepsilon_{n}^{2}$, (iii) $0<\beta_{n+1}-\gamma_{n+1}<\varepsilon_{n}^{2}$ and (iv) $\gamma_{n+1}-\gamma_{n}>0$.

From (ii) and (iii), we can obtain that $\varepsilon_{n+1}=\alpha_{n+1}-\gamma_{n+1}<2 \varepsilon_{n}^{2}<\frac{2}{81}$ for all positive integer $n$ by induction. It follows that for any positive integer $m \leq n$,

$$
\varepsilon_{n}<2 \varepsilon_{n-1}^{2}<2\left[2 \varepsilon_{n-2}^{2}\right]^{2}<\cdots<\frac{1}{2}\left[2 \varepsilon_{m}\right]^{2^{n-m}} .
$$

Since $\varepsilon_{m} \in\left(0, \frac{1}{9}\right)$ for any positive integer $m$, we have that the values of $\alpha_{n}, \beta_{n}, \gamma_{n}$ are close to each other when $n$ becomes large.

Numerically, we can find

$$
\lim _{n \rightarrow \infty} \alpha_{n}=\lim _{n \rightarrow \infty} \beta_{n}=\lim _{n \rightarrow \infty} \gamma_{n}=0.4135272769487595999 \ldots
$$

From the lemmas above, we get the bounds for the number of independent sets.

Theorem 3.3. For any positive integer $m \leq n$,

$$
f_{m}^{3^{n-m}}\left(1+2 \gamma_{m}\right)^{\frac{3\left(3^{n-m}-1\right)}{2}}\left(1+\gamma_{n}\right)^{3}<i_{n}<f_{m}^{3^{n-m}}\left(1+2 \alpha_{m}\right)^{\frac{3\left(3^{n-m}-1\right)}{2}}\left(1+\alpha_{n}\right)^{3}
$$

Proof. By Lemmas 3.1 and 3.2 and the definition of $\alpha_{n}, \beta_{n}, \gamma_{n}$, we have

$$
\begin{aligned}
f_{n} & =f_{n-1}^{3}\left(1+6 \alpha_{n-1}+3 \alpha_{n-1} \beta_{n-1}+9 \alpha_{n-1}^{2}+6 \alpha_{n-1}^{2} \beta_{n-1}+2 \alpha_{n-1}^{3}\right) \\
& <f_{n-1}^{3}\left(1+6 \alpha_{n-1}+12 \alpha_{n-1}^{2}+8 \alpha_{n-1}^{3}\right) \\
& =\left[f_{n-1}\left(1+2 \alpha_{n-1}\right)\right]^{3}<\left[f_{n-2}^{3}\left(1+2 \alpha_{n-2}\right)^{3}\right]^{3}\left(1+2 \alpha_{n-1}\right)^{3} \\
& <f_{n-2}^{3^{2}}\left(1+2 \alpha_{n-2}\right)^{3^{2}+3^{1}} \\
& <\cdots<f_{m}^{3^{n-m}}\left(1+2 \alpha_{m}\right)^{\frac{3\left(3^{n-m}-1\right)}{2}} .
\end{aligned}
$$

And

$$
\begin{aligned}
i_{n} & =f_{n}+3 g_{n}+3 h_{n}+p_{n}=f_{n}\left(1+3 \alpha_{n}+3 \alpha_{n} \beta_{n}+\alpha_{n} \beta_{n} \gamma_{n}\right) \\
& <f_{n}\left(1+3 \alpha_{n}+3 \alpha_{n}^{2}+\alpha_{n}^{3}\right)=f_{n}\left(1+\alpha_{n}\right)^{3}<f_{m}^{3^{n-m}}\left(1+2 \alpha_{m}\right)^{\frac{3\left(3^{n-m}-1\right)}{2}}\left(1+\alpha_{n}\right)^{3} .
\end{aligned}
$$

Similarly, the lower bound for $i_{n}$ can be derived.

Theorem 3.4. The asymptotic growth constant for the number of independent sets in $H_{n}$ is bounded by

$$
\frac{\ln f_{m}}{3^{m+1}}+\frac{\ln \left(1+2 \gamma_{m}\right)}{2 \times 3^{m}} \leq \mu \leq \frac{\ln f_{m}}{3^{m+1}}+\frac{\ln \left(1+2 \alpha_{m}\right)}{2 \times 3^{m}}
$$

where $m$ is a positive integer. 
Proof. Note that the number of vertices of $H_{n}$ is $v\left(H_{n}\right)=3^{n+1}$, by Theorem 3.3, we have

$$
\frac{\ln i_{n}}{v\left(H_{n}\right)}<\frac{\ln f_{m}}{3^{m+1}}+\frac{\ln \left(1+2 \alpha_{m}\right)}{2 \times 3^{m}}-\frac{\ln \left(1+2 \alpha_{m}\right)}{2 \times 3^{n}}+\frac{\ln \left(1+\alpha_{n}\right)}{3^{n}}
$$

and

$$
\frac{\ln i_{n}}{v\left(H_{n}\right)}>\frac{\ln f_{m}}{3^{m+1}}+\frac{\ln \left(1+2 \gamma_{m}\right)}{2 \times 3^{m}}-\frac{\ln \left(1+2 \gamma_{m}\right)}{2 \times 3^{n}}+\frac{\ln \left(1+\gamma_{n}\right)}{3^{n}}
$$

So, the bounds for $\mu=\lim _{v\left(H_{n}\right) \rightarrow \infty} \frac{\ln i_{n}}{v\left(H_{n}\right)}$ follow.

As $m$ increases, the difference between the upper and lower bounder in Theorem 3.4 becomes small and the convergence is rapid. Numerically, the asymptotic growth constant for the number of independent sets of the Tower of Hanoi graph $H_{n}$ in the large $n$ limit is $\mu=0.42433435855938823 \cdots$. In fact, the numerical value of $\mu$ can be obtained with more than a hundred significant figures accurate when $m$ is equal to seven.

\section{The number of independent sets on graphs $S_{k, n}$}

The Sierpiński graphs $S_{k, n}$ were introduced by Klavžar and Milutinović in 1997 in [25]. The graph $S_{k, 0}$ is simply the complete graph on $k$ vertices, $S_{k, n}$ is constructed from $S_{k, n-1}$ by copying $k$ times $S_{k, n-1}$ and adding exactly one edge between each pair of copies. For the construction, one can easily derive that the total number of vertices and edges in $S_{k, n}$ are $v\left(S_{k, n}\right)=k^{n+1}$ and $e\left(S_{k, n}\right)=\frac{1}{2}\left(k^{n+2}-k\right)$, respectively. In particularly, we can see those graphs are exactly the graphs of the Tower of Hanoi problem for $k=3$ and another case as shown in Figure 6 for $k=4$.
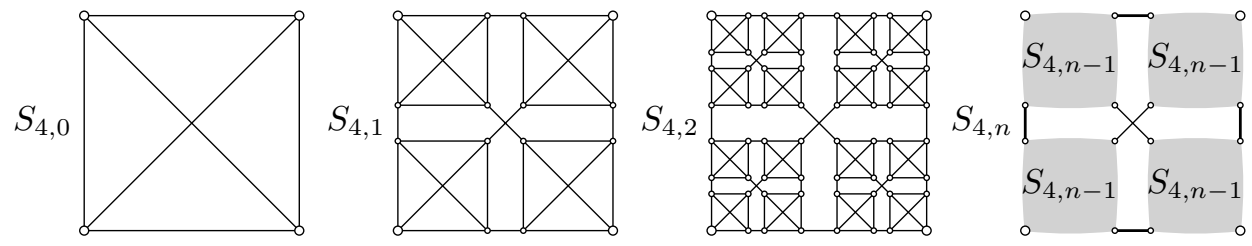

Figure 6: The graphs $S_{4,0}, S_{4,1}, S_{4,2}$ and the construction of $S_{4, n}$.

The method given in the previous section can be applied to enumeration the number of independent sets on this Sierpiński graphs with $k \geq 4$, but the items of the recursion relations will become larger and larger with the increase of $k$.

To seek the number of independent sets on $S_{4, n}$, we use the following definitions: (i) Define $f_{4, n}$ as the number of independent sets such that all four outmost vertices are not in the vertex sets. (ii) Define $g_{4, n}$ as the number of independent sets such that only one certain outmost vertex are in the vertex sets. (iii) Define $h_{4, n}$ as the number of independent sets such that exactly two certain outmost vertex are in the vertex sets. (iv) Define $p_{4, n}$ as the number of independent sets such that exactly three certain outmost vertex are in the vertex sets. (v) Define $q_{4, n}$ as the number of independent sets such that all four outmost vertex are in the vertex sets. 
Table 3: The first few values of $f_{4, n}, g_{4, n}, h_{4, n}, p_{4, n}, q_{4, n}$ and $i_{4, n}$ on $S_{4, n}$.

\begin{tabular}{rrrr}
\hline$n$ & 1 & 2 & 3 \\
\hline$f_{4, n}$ & 163 & 13064274739 & 497661511371512614009322138806617451967507 \\
$g_{4, n}$ & 52 & 3951119257 & 150487045809089786329485928937399858428184 \\
$h_{4, n}$ & 15 & 1194624638 & 45505530112368879421817904248654649805971 \\
$p_{4, n}$ & 4 & 361093492 & 13760342318790991781550553074012255470504 \\
$q_{4, n}$ & 1 & 109115158 & 4160967243331065589513567798163834387921 \\
$i_{4, n}$ & 478 & 37589988721 & 1431845211800580068573889060142357640786006 \\
\hline
\end{tabular}

Table 4: The first few values of $\alpha_{4, n}, \beta_{4, n}, \gamma_{4, n}$ and $\delta_{4, n}$ on $S_{4, n}$.

\begin{tabular}{rrrr}
\hline$n$ & 1 & 2 & 3 \\
\hline$\alpha_{4, n}$ & 0.319018404907975 & 0.302436938592921 & 0.302388355077651 \\
$\beta_{4, n}$ & 0.288461538461538 & 0.302350944199809 & 0.302388354211550 \\
$\gamma_{4, n}$ & 0.266666666666666 & 0.302265230863252 & 0.302388353345449 \\
$\delta_{4, n}$ & 0.25 & 0.302179796693760 & 0.302388352479348 \\
\hline
\end{tabular}

The quantities $f_{4, n}, g_{4, n}, h_{4, n}, p_{4, n}, q_{4, n}$ of $S_{4, n}$ are lengthy and given in the appendix. Some values of $f_{4, n}, g_{4, n}, h_{4, n}, p_{4, n}, q_{4, n}, i_{4, n}$ are listed in Table 3 . These numbers grow exponentially, and have no integer factorizations. There are four equivalent $g_{4, n}$, six equivalent $h_{4, n}$, and four equivalent $p_{n}$. By definition, we have

$$
i_{4, n}=f_{4, n}+4 g_{4, n}+6 h_{4, n}+4 p_{4, n}+q_{4, n} .
$$

The initial values at stage zero are $f_{4,0}=g_{4,0}=1, h_{4,0}=p_{4,0}=q_{4,0}=0$ and $i_{4,0}=5$.

Define ratios $\alpha_{4, n}=g_{4, n} / f_{4, n}, \beta_{4, n}=h_{4, n} / g_{4, n}, \gamma_{4, n}=p_{4, n} / h_{4, n}, \delta_{4, n}=q_{4, n} / p_{4, n}$. As $n$ increases, we find $\alpha_{4, n}$ decrease monotonically while $\beta_{4, n}, \gamma_{4, n}$ and $\delta_{4, n}$ increase monotonically with the relation $\alpha_{4, n}>\beta_{4, n}>\gamma_{4, n}>\delta_{4, n}$. The values of these ratios for small $n$ are listed in Table 4. Numerically, we can find

$$
\lim _{n \rightarrow \infty} \alpha_{4, n}=\lim _{n \rightarrow \infty} \beta_{4, n}=\lim _{n \rightarrow \infty} \gamma_{4, n}=\lim _{n \rightarrow \infty} \delta_{4, n}=0.30238835458805297767 \ldots
$$

By a similar argument as the Tower of Hanoi graph $H_{n}$ in the last section, the asymptotic growth constant for the number of independent sets on $S_{4, n}$ is bounded by

$$
\frac{\ln f_{4, m}}{4^{m+1}}+\frac{\ln \left(1+2 \delta_{4, m}\right)}{2 \times 4^{m}} \leq \mu_{4} \leq \frac{\ln f_{4, m}}{4^{m+1}}+\frac{\ln \left(1+2 \alpha_{4, m}\right)}{2 \times 4^{m}}
$$

where $\mu_{4}=\lim _{v\left(S_{4, n}\right) \rightarrow \infty} \frac{\ln i_{4, n}}{v\left(S_{4, n}\right)}$ and $m$ is a positive integer.

Then, we can obtain the asymptotic growth constant for the number of independent sets on the Sierpińsk graph $S_{4, n}$ in the large $n$ limit is $\mu=0.378737140730676994823835 \cdots$.

We can also continue verify a similarly bound for the asymptotic growth constant on $S_{5, n}$, in order to avoid verbosity, we are not to describe here. However, the recursion relations of the number of independent sets for general $k$ are difficult to obtain. From what has been discussed above, we have the following conjecture for the Sierpiński graphs $S_{k, n}$ with positive integers $k$ and $m$. 
Conjecture 4.1. The asymptotic growth constant for the number of independent sets on the Sierpinsk graph $S_{4, n}$ is bounded by

$$
\frac{\ln f_{k, m}}{k^{m+1}}+\frac{\ln \left(1+2 \phi_{k, m}\right)}{2 \times k^{m}} \leq \mu_{k} \leq \frac{\ln f_{k, m}}{k^{m+1}}+\frac{\ln \left(1+2 \alpha_{k, m}\right)}{2 \times k^{m}}
$$

where the ratios are defined as $\alpha_{k, n}=g_{k, n} / f_{k, n}, \phi_{k, n}=w_{k, n} / y_{k, n}, f_{k, n}$ is the number of independent sets such that all $k$ outmost vertices are not in the vertex subset, $g_{k, n}$ is the number of independent sets such that one certain outmost vertex is in the vertex subset, $y_{k, n}$ is number of independent sets such that all but one certain outmost vertex are in the vertex subset, and $w_{k, n}$ is the number of independent sets such that all $k$ outmost vertices are in the vertex subset.

\section{Appendix: Recursion relation for $S_{4, n}$}

We give the recursive relation for the Siepiński graph $S_{4, n}$ here. Since the subscript is $k=4$ for all the quantities throughout this section, we will use the simplified notation $f_{n+1}$ to denote $f_{4, n+1}$ and similar notations for other quantities. For any non-negative integer $n$, we have

$$
f_{n+1}=f_{n}^{4}+12 f_{n}^{3} g_{n}+12 f_{n}^{3} h_{n}+48 f_{n}^{2} g_{n}^{2}+4 f_{n}^{3} p_{n}+84 f_{n}^{2} g_{n} h_{n}+72 f_{n} g_{n}^{3}+24 f_{n}^{2} g_{n} p_{n}+
$$
$30 f_{n}^{2} h_{n}^{2}+156 f_{n} g_{n}^{2} h_{n}+30 g_{n}^{4}+12 f_{n}^{2} h_{n} p_{n}+36 f_{n} g_{n}^{2} p_{n}+84 f_{n} g_{n} h_{n}^{2}+60 g_{n}^{3} h_{n}+$ $24 f_{n} g_{n} h_{n} p_{n}+8 g_{n}^{3} p_{n}+8 f_{n} h_{n}^{3}+24 g_{n}^{2} h_{n}^{2}$,

$$
g_{n+1}=f_{n}^{3} g_{n}+3 f_{n}^{3} h_{n}+9 f_{n}^{2} g_{n}^{2}+3 f_{n}^{3} p_{n}+33 f_{n}^{2} g_{n} h_{n}+24 f_{n} g_{n}^{3}+f_{n}^{3} q_{n}+24 f_{n}^{2} g_{n} p_{n}+
$$
$21 f_{n}^{2} h_{n}^{2}+96 f_{n} g_{n}^{2} h_{n}+18 g_{n}^{4}+6 f_{n}^{2} g_{n} q_{n}+21 f_{n}^{2} h_{n} p_{n}+51 f_{n} g_{n}^{2} p_{n}+93 f_{n} g_{n} h_{n}^{2}+69 g_{n}^{3} h_{n}+$ $3 f_{n}^{2} h_{n} q_{n}+9 f_{n} g_{n}^{2} q_{n}+3 f_{n}^{2} p_{n}^{2}+66 f_{n} g_{n} h_{n} p_{n}+24 g_{n}^{3} p_{n}+21 f_{n} h_{n}^{3}+66 g_{n}^{2} h_{n}^{2}+6 f_{n} g_{n} h_{n} q_{n}+$ $2 g_{n}^{3} q_{n}+6 f_{n} g_{n} p_{n}^{2}+12 f_{n} h_{n}^{2} p_{n}+24 g_{n}^{2} h_{n} p_{n}+14 g_{n} h_{n}^{3}$,

$$
h_{n+1}=f_{n}^{2} g_{n}^{2}+6 f_{n}^{2} g_{n} h_{n}+6 f_{n} g_{n}^{3}+6 f_{n}^{2} g_{n} p_{n}+8 f_{n}^{2} h_{n}^{2}+38 f_{n} g_{n}^{2} h_{n}+8 g_{n}^{4}+2 f_{n}^{2} g_{n} q_{n}+
$$
$14 f_{n}^{2} h_{n} p_{n}+30 f_{n} g_{n}^{2} p_{n}+64 f_{n} g_{n} h_{n}^{2}+50 g_{n}^{3} h_{n}+4 f_{n}^{2} h_{n} q_{n}+8 f_{n} g_{n}^{2} q_{n}+5 f_{n}^{2} p_{n}^{2}+$ $80 f_{n} g_{n} h_{n} p_{n}+30 g_{n}^{3} p_{n}+26 f_{n} h_{n}^{3}+87 g_{n}^{2} h_{n}^{2}+2 f_{n}^{2} p_{n} q_{n}+16 f_{n} g_{n} h_{n} q_{n}+6 g_{n}^{3} q_{n}+$ $18 f_{n} g_{n} p_{n}^{2}+34 f_{n} h_{n}^{2} p_{n}+72 g_{n}^{2} h_{n} p_{n}+44 g_{n} h_{n}^{3}+4 f_{n} g_{n} p_{n} q_{n}+4 f_{n} h_{n}^{2} q_{n}+8 g_{n}^{2} h_{n} q_{n}+$ $8 f_{n} h_{n} p_{n}^{2}+8 g_{n}^{2} p_{n}^{2}+28 g_{n} h_{n}^{2} p_{n}+4 h_{n}^{4}$,

$$
p_{n+1}=f_{n} g_{n}^{3}+9 f_{n} g_{n}^{2} h_{n}+3 g_{n}^{4}+9 f_{n} g_{n}^{2} p_{n}+24 f_{n} g_{n} h_{n}^{2}+27 g_{n}^{3} h_{n}+3 f_{n} g_{n}^{2} q_{n}+
$$
$42 f_{n} g_{n} h_{n} p_{n}+22 g_{n}^{3} p_{n}+18 f_{n} h_{n}^{3}+75 g_{n}^{2} h_{n}^{2}+12 f_{n} g_{n} h_{n} q_{n}+6 g_{n}^{3} q_{n}+15 f_{n} g_{n} p_{n}^{2}+$ $39 f_{n} h_{n}^{2} p_{n}+99 g_{n}^{2} h_{n} p_{n}+69 g_{n} h_{n}^{3}+6 f_{n} g_{n} p_{n} q_{n}+9 f_{n} h_{n}^{2} q_{n}+21 g_{n}^{2} h_{n} q_{n}+21 f_{n} h_{n} p_{n}^{2}+$ $24 g_{n}^{2} p_{n}^{2}+96 g_{n} h_{n}^{2} p_{n}+15 h_{n}^{4}+6 f_{n} h_{n} p_{n} q_{n}+6 g_{n}^{2} p_{n} q_{n}+12 g_{n} h_{n}^{2} q_{n}+2 f_{n} p_{n}^{3}+24 g_{n} h_{n} p_{n}^{2}+$ $14 h_{n}^{3} p_{n}$,

$$
\begin{aligned}
& q_{n+1}=g_{n}^{4}+12 g_{n}^{3} h_{n}+12 g_{n}^{3} p_{n}+48 g_{n}^{2} h_{n}^{2}+4 g_{n}^{3} q_{n}+84 g_{n}^{2} h_{n} p_{n}+72 g_{n} h_{n}^{3}+24 g_{n}^{2} h_{n} q_{n}+ \\
& 30 g_{n}^{2} p_{n}^{2}+156 g_{n} h_{n}^{2} p_{n}+30 h_{n}^{4}+12 g_{n}^{2} p_{n} q_{n}+36 g_{n} h_{n}^{2} q_{n}+84 g_{n} h_{n} p_{n}^{2}+60 h_{n}^{3} p_{n}+ \\
& 24 g_{n} h_{n} p_{n} q_{n}+8 h_{n}^{3} q_{n}+8 g_{n} p_{n}^{3}+24 h_{n}^{2} p_{n}^{2} .
\end{aligned}
$$

There are always $729=3^{6}$ terms in these equations. 


\section{Acknowledgments}

The authors are thankful to the anonymous referees for their useful comments.

\section{References}

[1] R. Baxter, I. Enting and S. Tsang, Hard-square lattice gas, J. Stat. Phys. 22 (1980), 465-489.

[2] B. Bollobás and B. D. McKay, The number of matchings in random regular graphs and bipartite graphs, J. Combin. Theory Ser. B 41 (1986), 80-91, doi:10.1016/0095-8956(86)90029-8, http://dx.doi.org/10.1016/0095-8956(86)90029-8.

[3] G. R. Brightwell, O. Häggström and P. Winkler, Nonmonotonic behavior in hard-core and widom-rowlinson models, J. Stat. Phys. 94 (1999), 415-435.

[4] N. J. Calkin and H. S. Wilf, The number of independent sets in a grid graph, SIAM J. Discrete Math. 11 (1998), 54-60 (electronic), doi:10.1137/S089548019528993X, http://dx . doi . org/10.1137/s089548019528993X.

[5] T.-H. Chan, A statistical analysis of the Towers of Hanoi problem, Int. J. Comput. Math. 28 (1989), 57-65, doi:10.1080/00207168908803728.

[6] S.-C. Chang, L.-C. Chen and W. Yan, Asymptotic enumeration of independent sets on the Sierpinski gasket, Filomat 27 (2013), 23-40, doi:10.2298/FIL1301023C, http: / / dx . doi. org/10.2298/FIL1301023C.

[7] H. Chen, R. Wu, G. Huang and H. Deng, Dimer-monomer model on the Towers of Hanoi graphs, Internat. J. Modern Phys. B 29 (2015), 1550173, 13, doi:10.1142/ S0217979215501738, http://dx.doi.org/10.1142/S0217979215501738.

[8] P. Cull and I. Nelson, Error-correcting codes on the Towers of Hanoi graphs, Discrete Math. 208/209 (1999), 157-175, doi:10.1016/S0012-365X(99)00070-9, combinatorics (Assisi, 1996), http://dx.doi.org/10.1016/s0012-365x(99)00070-9.

[9] K. Engel, On the Fibonacci number of an $m \times n$ lattice, Fibonacci Quart. 28 (1990), 72-78.

[10] E. J. Farrell, Counting matchings in graphs, J. Franklin Inst. 324 (1987), 331-339, doi: 10.1016/0016-0032(87)90046-9, http://dx.doi.org/10.1016/0016-0032 (87) $90046-9$.

[11] E. J. Farrell, Matchings in rectangular cacti, J. Math. Sci. (Calcutta) 9 (1998), 163-183.

[12] R. Grigorchuk and Z. Šunik, Asymptotic aspects of Schreier graphs and Hanoi Towers groups, C. R. Math. Acad. Sci. Paris 342 (2006), 545-550, doi:10.1016/j.crma.2006.02.001, http: //dx.doi.org/10.1016/j.crma.2006.02.001.

[13] W. Guo and H. W. Blöte, Finite-size analysis of the hard-square lattice gas, Physical Review E 66 (2002), 046140.

[14] I. Gutman and F. Harary, Generalizations of the matching polynomial, Utilitas Math. 24 (1983), 97-106.

[15] O. Häggström, Ergodicity of the hard-core model on $\mathbf{Z}^{2}$ with parity-dependent activities, Ark. Mat. 35 (1997), 171-184, doi:10.1007/BF02559597, http://dx.doi.org/10.1007/ BE02559597.

[16] J. R. Heringa, H. W. J. Blöte and E. Luijten, High-dimensional lattice gases, J. Phys. A 33 (2000), 2929-2941, doi:10.1088/0305-4470/33/15/302, http: / /dx. doi.org/ 10 . $1088 / 0305-4470 / 33 / 15 / 302$.

[17] A. M. Hinz, The Tower of Hanoi, Enseign. Math. (2) 35 (1989), 289-321. 
[18] A. M. Hinz, Pascal's triangle and the Tower of Hanoi, Amer. Math. Monthly 99 (1992), 538 544, doi:10.2307/2324061, http://dx.doi.org/10.2307/2324061.

[19] A. M. Hinz, Shortest paths between regular states of the Tower of Hanoi, Inform. Sci. 63 (1992), 173-181, doi:10.1016/0020-0255(92)90067-I, http://dx.doi .org/10 . 1016 / $0020-0255$ (92) $90067-I$.

[20] A. M. Hinz, S. Klavžar, U. Milutinović, D. Parisse and C. Petr, Metric properties of the Tower of Hanoi graphs and Stern's diatomic sequence, European J. Combin. 26 (2005), 693-708, doi: 10.1016/j.ejc.2004.04.009, http://dx.doi.org/10.1016/j.ejc.2004.04.009.

[21] A. M. Hinz, S. Klavžar, U. Milutinović and C. Petr, The tower of Hanoi-myths and maths, Birkhäuser/Springer Basel AG, Basel, 2013, doi:10.1007/978-3-0348-0237-6, with a foreword by Ian Stewart, http://dx.doi.org/10.1007/978-3-0348-0237-6.

[22] A. M. Hinz and D. Parisse, On the planarity of Hanoi graphs, Expo. Math. 20 (2002), 263-268, doi:10.1016/S0723-0869(02)80023-8, http://dx. doi.org/10 . 1016/S0723-0869(02)80023-8.

[23] H. Hosoya, Topological index. a newly proposed quantity characterizing the topological nature of structural isomers of saturated hydrocarbons, Bull. Chem. Soc. Jpn. 44 (1971), 2332-2339.

[24] J. Kahn, An entropy approach to the hard-core model on bipartite graphs, Combin. Probab. Comput. 10 (2001), 219-237, doi:10.1017/S0963548301004631, http:// dx.doi.org/ $10.1017 /$ S0963548301004631.

[25] S. Klavžar and U. Milutinović, Graphs $S(n, k)$ and a variant of the Tower of Hanoi problem, Czechoslovak Math. J. 47(122) (1997), 95-104, doi:10.1023/A:1022444205860, http:// dx.doi.org/10.1023/A:1022444205860.

[26] M. Klazar, Twelve countings with rooted plane trees, European J. Combin. 18 (1997), 195-210, doi:10.1006/eujc.1995.0095, http://dx.doi.org/10.1006/eujc.1995.0095.

[27] H.-F. Law, On the number of independent sets in a tree, Electron. J. Combin. 17 (2010), Note 18, 5, http://www.combinatorics.org/Volume_17/Abstracts/v17iln18. html.

[28] C.-K. Li and I. Nelson, Perfect codes on the Towers of Hanoi graph, Bull. Austral. Math. Soc. 57 (1998), 367-376, doi:10.1017/S0004972700031774, http://dx. doi.org/10. $1017 / \mathrm{S} 0004972700031774$.

[29] V. Linek, Bipartite graphs can have any number of independent sets, Discrete Math. 76 (1989), 131-136, doi:10.1016/0012-365X(89)90306-3, http://dx.doi.org/10. $1016 / 0012-365 \times(89) 90306-3$.

[30] R. E. Merrifield and H. E. Simmons, Topological methods in chemistry, Wiley New York etc., 1989.

[31] D. Romik, Shortest paths in the Tower of Hanoi graph and finite automata, SIAM J. Discrete Math. 20 (2006), 610-622 (electronic), doi:10.1137/050628660, http://dx.doi.org/ $10.1137 / 050628660$.

[32] D. H. Rouvray, The role of graph-theoretical invariants in chemistry, in: Proceedings of the seventeenth Southeastern international conference on combinatorics, graph theory, and computing (Boca Raton, Fla., 1986), volume 55, 1986 pp. 253-265.

[33] L. K. Runnels, Phase transitions of hard sphere lattice gases, Comm. Math. Phys. 40 (1975), $37-48$.

[34] A. D. Scott and A. D. Sokal, The repulsive lattice gas, the independent-set polynomial, and the Lovász local lemma, J. Stat. Phys. 118 (2005), 1151-1261, doi:10.1007/s10955-004-2055-4, http://dx.doi.org/10.1007/s10955-004-2055-4. 
[35] E. Teufl and S. Wagner, Enumeration problems for classes of self-similar graphs, J. Combin. Theory Ser. A 114 (2007), 1254-1277, doi:10.1016/j.jcta.2007.01.007, http://dx.doi. org/10.1016/j.jcta.2007.01.007.

[36] E. Teufl and S. Wagner, Exact and asymptotic enumeration of perfect matchings in self-similar graphs, Discrete Math. 309 (2009), 6612-6625, doi:10.1016/j.disc.2009.07.009, http:// dx.doi.org/10.1016/j.disc.2009.07.009.

[37] E. Teufl and S. Wagner, Resistance scaling and the number of spanning trees in self-similar lattices, J. Stat. Phys. 142 (2011), 879-897, doi:10.1007/s10955-011-0140-z, http: / dx . doi.org/10.1007/s10955-011-0140-z.

[38] N. Trinajstić, Chemical graph theory, Mathematical Chemistry Series, CRC Press, Boca Raton, FL, 2nd edition, 1992, doi:10.1007/s10910-008-9464-6, http: / / dx. doi.org/10 . $1007 / \mathrm{s} 10910-008-9464-6$.

[39] J. van den Berg and J. E. Steif, Percolation and the hard-core lattice gas model, Stochastic Process. Appl. 49 (1994), 179-197, doi:10.1016/0304-4149(94)90132-5, http: / / dx . doi. org/10.1016/0304-4149(94)90132-5.

[40] K. Weber, On the number of stable sets in an $m \times n$ lattice, Rostock. Math. Kolloq. (1988), 28-36.

[41] Y. Zhao, The number of independent sets in a regular graph, Combin. Probab. Comput. 19 (2010), 315-320, doi:10.1017/S0963548309990538, http://dx.doi.org/10.1017/ S0963548309990538. 